УДК 621.396

\title{
КОНЦЕПЦИЯ ФИЗИЧЕСКОГО УРОВНЯ СИСТЕМ СВЯЗИ ПЯТОГО ПОКОЛЕНИЯ
}

\author{
Д. А. ПОКАМЕСТОВ, Я. В. КРЮКОВ, Е. В. РОГОЖНИКОВ, Р. Р. АБЕНОВ, А. Я. ДЕМИДОВ
}

Томский государственный университет систем управления и радиоэлектроники, Россия, Томск, 634050, пр-т Ленина, 40

\begin{abstract}
Аннотация. Рассмотрены наиболее перспективные с точки зрения использования частотно-временного ресурса технологии формирования сигналов и множественного доступа: технология полного дуплекса, метод формирования поднесущих с помощью банка гребенчатых фильтров, метод неортогонального множественного доступа и метод множественного доступа с разреженным кодом. Описаны основные существующие схемы реализации полнодуплексной связи, основанные на аналоговой и цифровой компенсации сигнала собственного передатчика в приемном канале. Рассмотрена система фильтрации многочастотного сигнала с помощью банка гребенчатых фильтров. Показано, что спектр такого сигнала имеет значительно меньшее внеполосное излучение по сравнению с существующими способами формирования ортогональных поднесущих. Исследован метод неортогонального множественного доступа и метод множественного доступа с разреженным кодом, приведены зависимости вероятностей битовых ошибок от отношения сигнал шум для систем связи, построенных с использованием этих методов. Показано, что рассматриваемые методы множественного доступа более эффективно используют частотно-временной ресурс по сравнению с существующими технологиями.
\end{abstract}

Ключевые слова: 5G; система связи пятого поколения; полный дуплекс; full duplex; аналоговая компенсация; цифровая компенсация; банк гребенчатых фильтров; FBMC; неортогональный множественный доступ; NOMA; SIC; множественный доступ с разреженным кодом; SCMA

\section{ВВЕДЕНИЕ}

В настоящее время продолжают развиваться системы передачи данных, в том числе мобильные сети беспроводного широкополосного доступа. Практически во всей зоне покрытия мобильных операторов развернуты сети четвертого поколения LTE. При этом объемы передаваемого трафика год от года увеличиваются, как и количество подключаемых к сети устройств. Если на момент разработки стандарта LTE это были в основном мобильные абонентские устройства, то сейчас все большую долю занимают различные датчики и «умные» устройства, что входит в концепцию «интернета вещей» IoT (internet of things).
В научных кругах идет обсуждение технического облика сетей связи пятого поколения. Крупнейшими компаниями, работающими в сфере электроники и телекоммуникаций, создан ряд проектов, в рамках которых идет работа по формированию концепции стандарта системы 5G. Из этих проектов стоит выделить группу METIS [1], созданную Европейской комиссией при поддержке Alcatel, Ericsson, Nokia, Huawei и др., работающую совместно с ней группу 5GPPP, немецкую команду NGMN [2], проект китайского правительства IMT-2020 (5G) Promotion Group [3], южнокорейский $5 \mathrm{G}$ Forum $[4,5]$. При этом в документах различных проектов рассматриваются схо- 
БИБЛИОГРАФИЧЕСКИЙ СПИСОК

1. Marsch, P.; Da Silva, I.; El Ayoubi, S. E.; Boldi, O. M.; et al. 5G RAN Architecture and Functional Design. METIS White Paper. 2016. 27 p.

2. 5G Initiative Team. NGMN 5G White Paper. 2015. $125 \mathrm{p}$.

3. 5G PPP Architecture Working Group. View on $5 \mathrm{G}$ Architecture (white paper). 2016.61 p.

4. 5G Network Architecture Design. IMT-2020 White Paper. 2016, 25 p.

5. Тихвинский, В. О.; Бочечка, Г. С. Концептуальные аспекты создания 5G. Электросвязь, № 10, С. 29-34, 2013. URL: http://w.raenitt.ru/publication/5G projects elsv.pdf.

6. Jain, M.; Choi, Jung Il; Kim, Taemin; Bharadia, Dinesh; Seth, Siddharth; Srinivasan, Kannan; Levis, Philip; Katti, Sachin; Sinha, Prasun. Practical, real-time, full duplex wireless. Proc. of 17th Annual Int. Conf. on Mobile Computing and Networking, ACM, 2011. C. 301-312. DOI: $\underline{10.1145 / 2030613.2030647 .}$.

7. Choi, Jung Il; Jain, Mayank; Srinivasan, Kannan; Levis, Phil; Katti, Sachin. Achieving single channel, full duplex wireless communication. Proc. of Sixteenth Annual Int. Conf. on Mobile Computing and Networking, ACM, 2010, pp. 1-12. DOI: 10.1145/1859995.1859997.

8. Duarte, M.; Dick, C.; Sabharwal, A. Experiment-driven characterization of full-duplex wireless systems. IEEE Trans. Wireless Commun., Vol. 11, No. 12, p. 4296-4307, 2012. DOI: 10.1109/TWC.2012.102612. $\underline{111278}$.

9. Rogozhnikov, E. V.; Koldomov, A. S.; Vorobyov, V. A. Full duplex wireless communication system, analog cancellation: Review of methods and experimental research. Proc. of XII Int. Siberian Conf. on Control and Communications, SIBCON-2016, 12-14 May 2016, Moscow, Russia. IEEE, 2016, p. 1-5. DOI: 10.1109/ SIBCON.2016.7491777.

10. Bharadia, Dinesh; McMilin, Emily; Katti, Sachin. Full duplex radios. Proc. of Conf. on SIGCOMM, 12-16 Aug. 2013, Hong Kong, China. Vol. 43, No. 4, p. 375-386. DOI: $10.1145 / 2534169.2486033$.

11. Абенов, Р.Р.; Вершинин, А.С.; Ворошилин, Е.П.; Рогожников, Е.В.Исследование методов эквалайзирования для систем связи с использованием OFDM сигналов. Вестник СибГУТИ, № 1, С. 50-56, 2013. URL: https://elibrary.ru/item.asp?id=19422242.

12. Каплун, Д.; Канатов, И.; Азаренков, Л.Банк цифровых фильтров. Компоненты и Технологии, № 
10, c. $156,2007$. URL: http://kit-e.ru/articles/dsp/2007 10 156.php.

13. Bellanger, M.; et al. FBMC physical layer: $a$ primer. PHYDYAS, Jan. 2010.

14. 5G Waveform Candidate Selection. URL: http://www.5gnow.eu/wp-content/uploads/2015/04/5GN OW D3.1 v1.1 final.pdf.

15. Premnath, S. N.; Wasden, D.; Kasera, S. K.; Patwari, N.; Farhang-Boroujeny, B. Beyond OFDM: Best-effort dynamic spectrum access using filterbank multicarrier. IEEE/ACM Trans. Networking, Vol. 21, No. 3, June 2013. DOI: 10.1109/TNET.2012.2213344.

16. Балашова, К. В.; Лобанов, Н. А.; Долгих, Д.А. Filter bank multicarrier модулятор. Матер. Всерос. науч.-техн. конф. студентов, аспирантов и молодых ученых «Научная сессия ТУСУР-2012», 16-18 мая 2012, Томск, Россия. Томск, 2012, Ч. 2, С. 75-78.

17. Farhang-Boroujeny, B. OFDM versus filter bank multicarrier. IEEE Signal Processing Magazine, Vol. 28, No. 3, P. 92-112, 2011. DOI: 10.1109/MSP.2011.940267.

18. Marcus, M. J. 5G and «IMT for 2020 and beyond» [Spectrum Policy and Regulatory Issues]. IEEE Wireless Commun., Vol. 22, No. 4, P. 2-3, 2015. DOI: 10.1109/MWC.2015.7224717.

19. Soldani, D.; Manzalini, A. Horizon 2020 and beyond: on the $5 \mathrm{G}$ operating system for a true digital society. IEEE Vehicular Technology Magazine, Vol. 10, No. 1, P. 32-42, 2015. DOI: 10.1109/MVT.2014.238 $\underline{0581 .}$.

20. Benjebbour, Anass; Saito, Keisuke; Li, Anxin; Kishiyama, Yoshihisa; Nakamura, Takehiro. Non-orthogonal multiple access (NOMA): Concept, performance evaluation and experimental trials. Proc. of Int. Conf. on Wireless Networks and Mobile Communications, WINCOM, 20-23 Oct. 2015, Marrakech, Morocco. IEEE, 2015, p. 1-6. DOI: 10.1109/ WINCOM.2015.7381343.

21. Zhang, Yi; Wang, Hui-Ming; Yang, Qian; Ding, Zhiguo. Secrecy sum rate maximization in non-orthogonal multiple access. IEEE Commun. Lett., Vol. 20, No. 5, P. 930-933, 2016. DOI: 10.1109/LCOMM.2016.2539162.
22. Dai, Linglong; Wang, Bichai; Yuan, Yifei; Han, Shuangfeng; I, Chih-lin; Wang, Zhaocheng. Non-orthogonal multiple access for 5G: solutions, challenges, opportunities, and future research trends. IEEE Commun. Magazine, Vol. 53, No. 9, P. 74-81, 2015. DOI: 10.1109/MCOM.2015.7263349.

23. Крюков, Я.В.; Демидов, А.Я.; Покаместов, Д.А. Алгоритм расчета мощности каналов при неортогональном множественном доступе NOMA. Доклады ТУСУР, Т. 19, № 4, С. 91-94, 2016. URL: https://journal.tusur.ru/ru/arhiv/4-2016/algoritm-rascheta -moschnosti-kanalov-pri-neortogonalnom-mnozhestvenn om-dostupe-noma.

24. 3GPP TS 36.211, version 9.1.0, 3rd Generation Partnership Project, Technical Specification Group Radio Access Network, Evolved Universal Terrestrial Radio Access (E-UTRA), Physical Channels and Modulation, 2010.

25. Nikopour, H.; Baligh, H. Sparse code multiple access. Proc. of 24th Int. Symp. on Personal Indoor and Mobile Radio Communications, PIMRC, 8-11 Sept. 2013, London, UK. IEEE, 2013, pp. 332-336. DOI: 10.1109/ PIMRC.2013.6666156.

26. Mu, Hang; Ma, Zheng; Alhaji, Mahamuda; Fan, Pingzhi; Chen, Dageng. A fixed low complexity message pass algorithm detector for up-link SCMA system. IEEE Wireless Commun. Lett., Vol. 4, No. 6, P. 585-588, 2015. DOI: $10.1109 /$ LWC.2015.2469668.

27. Покаместов, Д.А.; Демидов, А.Я.; Крюков, Я. В.; Рогожников, Е. В.; Абенов, Р. Р. Формирование и обработка сигналов множественного доступа с разреженным кодом. Электросвязь, № 10, С. 56-61, 2016.

28. Покаместов, Д.А.; Демидов, А.Я.; Крюков, Я.В.Влияние формирующих матриц на помехозащищенность каналов связи с множественным доступом на основе разреженных кодов. Доклады ТУСУР, Т. 19, № 3, C. 65-69, 2016. URL: https://journal.tusur. ru/ru/arhiv/3-2016/vliyanie-formiruyuschih-matrits-na-p omehozaschischennost-kanalov-svyazi-s-mnozhestvenny m-dostupom-na-osnove-razrezhennyh-kodov. 